\title{
IMPACTO ECONOMICO Y SOCIAL DE LA RESOLUCIÓN NO. 1771 DEL 28 DE OCTUBRE 2016 (DELIMITACION DE PARAMOS) DEL MINISTERIO DE AMBIENTE Y DESARROLLO SOSTENIBLE EN LA POBLACION DEL MUNICIPIO DE AQUITANIA
}

Oscar Darío Hurtado Pérez

Julián David Mariño Sosa

\begin{abstract}
RESUMEN
La población del Municipio de Aquitania desarrolla sus actividades económicas de agricultura y ganadería en una amplia zona terrestre, gran parte de la cual fue delimitada como páramo de la cuenca Lago de Tota mediante la resolución No. 1771 del 28 de Octubre 2016 del Ministerio de Ambiente y Desarrollo Sostenible, lo cual genero una gran problemática para el sector poblacional que desarrolla sus principales actividades económicas allí y que según lo que se estableció en dicha resolución ya no podrá seguir realizando las actividades acostumbradas, dadas las nuevas condiciones legales bajo las que se rigen estos terrenos. Aun cuando por medio de este tipo de delimitaciones el gobierno busca la conservación de los páramos y del medio ambiente, la realidad es que la aplicación de la resolución en mención trajo consigo varios inconvenientes a resolver, ya que aquellas familias que durante décadas desarrollan sus principales actividades económicas allí se ven afectadas por lo reglamentado. En su mayoría dicho sector poblacional está conformado por los campesinos y habitantes oriundos de la región, para quienes la agricultura y ganadería
\end{abstract}


son las únicas actividades de sustento que conocen y de las que a su vez, depende el sustento de varias familias.

En esta investigación se determinaron los impactos económicos y sociales generados por la resolución anteriormente mencionada en la población del Municipio de Aquitania y se plantearon acciones necesarias para la mitigación de estas problemáticas.

PALABRAS CLAVE: Aquitania, CorpoBoyacá, Ministerio del Medio Ambiente y Desarrollo Sostenible, Delimitación de Páramos, Agricultura.

\begin{abstract}
The problem of the delimitation of paramos affects the families that carry out agricultural and livestock activities in these lands. Even though, the reason to be of this type of delimitations is the intention from the government to seeks the conservation of the paramos and the environment, the reality is that the application of the resolution in question brought with it several inconveniences to be solved, since those families that for decades develop their main economic activities there are affected by the regulations. In its majority, this population sector is made up of the peasants and inhabitants of the region, for whom agriculture and livestock are the only livelihood activities they know and which, in turn, depends on the livelihood of several families.
\end{abstract}


SUMARIO: INTRODUCCIÓN, METODOLOGÍA, I) EXPOSICIÓN DE MOTIVOS DE LA RESOLUCIÓN NO. 1771 DEL 28 DE OCTUBRE 2016 DEL MINISTERIO DE

AMBIENTE Y DESARROLLO SOSTENIBLE, ii) REALIDAD ECONÓMICA Y

SOCIAL DEL MUNICIPIO DE AQUITANIA FRENTE A LAS RECOMENDACIONES

PARA LA DELIMITACIÓN DE PÁRAMOS III) CONCLUSIONES Y

RECOMENDACIONES, IV) REFERENCIAS

\section{INTRODUCCCIÓN}

Es claro que durante los últimos años en nuestro país se ha implementado una política ambiental dirigida a la conservación de los recursos naturales, esto de acuerdo a lo estipulado en los tratados internaciones ratificados por el estado colombiano y a las peticiones de la ciudadanía quienes cada vez se ven más involucrados en los temas ambientales y en la promoción de políticas ambientales dirigidas a la protección de los páramos. Lo anterior, teniendo en cuenta la ola capitalista que rige la economía mundial resulta innegablemente en un balance positivo para la conservación de los recursos naturales, pero aun así es inevitable que la implementación de dichas políticas genere consecuencias negativas en otros aspectos igualmente relevantes para la comunidad. En este caso en especifico, la población de Aquitania desarrolla actividades de agricultura llevadas a cabo por familias productoras durante décadas, actividades de las cuales se genera el sustento para estas familias y quienes se han dedicado durante años a trabajar la tierra de manera responsable. La delimitación que se dio por medio de la Resolución No. 1771 del 28 de Octubre 2016 del Ministerio de Ambiente y Desarrollo Sostenible genera un inconveniente, ya que al delimitar varios de los terrenos cercanos a los páramos y limitar 
las actividades que dichas familias desarrollaban allí, los productores campesinos se ven privados del único trabajo que conocen. Esta investigación busca abordar los impactos económicos y sociales que se generaron en la población del Municipio de Aquitania en consecuencia de la aplicación de la resolución No. 1771 del 28 de Octubre 2016 del Ministerio de Ambiente y Desarrollo Sostenible por medio de la cual se regula la delimitación de paramos, por medio de este artículo de busca determinar los entes competentes para la generación de las soluciones pertinentes y las acciones necesarias para la mitigación de estas problemáticas. Se hace necesario generar una hoja de ruta o acciones por parte de la administración municipal en cuanto a mitigar los daños económicos y sociales que se presentan en estas familias. Esta investigación busca servir como referencia para Municipios con similares condiciones geográficas, económicas y sociales que se encuentren en área de paramos, por lo anterior, se formuló como pregunta ¿Cuál es el Impacto económico y social de la resolución No. 1771 del 28 de Octubre 2016 del Ministerio de Ambiente y Desarrollo Sostenible en la población del Municipio de Aquitania? Para resolver ese interrogante se planteó como acción determinar el impacto económico y social de dicha resolución.

Ahora, para desarrollarlo se tuvieron tres momentos i) Analizar la exposición de motivos de la resolución No. 1771 del 28 de Octubre 2016 del Ministerio de Ambiente y Desarrollo Sostenible mediante la cual se delimito el páramo de la cuenca lago de tota, luego, de realizar la anterior acción ii) se determinó si las recomendaciones para la delimitación de Páramos se ajustan a la realidad económica y social del Municipio de Aquitania, para finalmente, iii) Generar conclusiones y recomendaciones a la administración municipal 
encaminadas a mitigar la problemática generada por la delimitación del páramo de la cuenca lago de tota en la población del Municipio de Aquitania.

\section{METODOLOGIA}

La metodología aplicada en la presente investigación fue de carácter documental, ya que se procedió a revisar los documentos públicos generados por entidades del orden nacional, departamental y municipal como el Ministerio de Ambiente y Desarrollo Sostenible, la Alcaldía de Aquitania, CorpoBoyacá entre otras entidades involucradas para la delimitación de paramos.

Por otro lado, se desarrolló una metodología descriptiva ya que con base en los documentos recopilados se evidenciaron las incidencias económicas y sociales en la población del Municipio de Aquitania y finalmente se desarrolló por medio de la aplicación del método analítico por medio del cual se logró determinar las estrategias que pueden ser implementadas por parte de la administración municipal para la mitigación de la problemática que se presentó.

\section{EXPOSICIÓN DE MOTIVOS DE LA RESOLUCIÓN NO. 1771 DEL 28 DE OCTUBRE 2016 DEL MINISTERIO DE AMBIENTE Y DESARROLLO SOSTENIBLE}

Dentro de la exposición de motivos de la resolución en mención se presenta el fundamento jurídico acerca de la delimitación de paramos, dicho tema no es nuevo para el estado 
colombiano, ya que desde hace más de dos décadas existen normas para su protección y conservación debido a que han sido reconocidos como áreas de especial importancia ecológica. (Min Ambiente, 2016).

Algunas de las normas que han venido tratando el tema de protección y conservación de paramos son las siguientes; Constitución Política de Colombia art. 8, 58, 79 y 80; Ley 99 de 1993 principio 4 articulo 1; Ley 1382 de 2010; Ley 1450 de 2011 articulo 202; Ley 1753 de 2015 articulo 173 y Sentencia Corte Constitucional C-035 de 2016, las cuales evidencian el orden cronológico de las leyes y normas que se han expedido por diferentes entidades de nivel nacional las cuales nos han llevado la Resolución 1771 del 28 de Octubre de 2016 de Ministerio de Ambiente y Desarrollo Sostenible por medio de la cual se expidió la delimitación del complejo de paramos Tota Bijagual - Mamapacha.

Cabe resaltar que mediante la Resolución 937 del 2011 del Ministerio de Ambiente y Desarrollo Sostenible: "se adoptó la cartografía 1:250.000 que el instituto Alexander Von Humboldt para la identificación y delimitación de los ecosistemas de paramos. La cual fue la cartografía usada por CORPOBOYACA para la delimitación del complejo de paramos”. (Min Ambiente, 2011).

El Ministrito de Ambiente y Desarrollo Sotenible afirma: “Que según lo estipulado en La ley 1450 de 2011: Por medio de la cual se expide el Plan Nacional de Desarrollo, 20102014, en su artículo 2, expone que los ecosistemas de paramos y humedales deberán ser delimitados a escala 1:25.000 con base en estudios técnicos, económicos, sociales y ambientales (ETESA), la delimitación será adoptada por dicha entidad mediante acto administrativo. Las Corporaciones Autónomas Regionales realizarán el proceso de zonificación, ordenamiento y determinación del régimen de usos de estos ecosistemas un 
plazo de hasta tres (3) años a partir de que se cuente con la delimitación, además no se podrán adelantar actividades agropecuarias, ni de exploración o explotación de hidrocarburos y minerales, ni construcción de refinerías de hidrocarburos.” (p.5).

Adicional a lo anterior, en el artículo 173 de dicha ley se expone que, el Ministerio de Ambiente y Desarrollo Sostenible hará la delimitación de las áreas de páramos en base a los estudios técnicos, ambientales, sociales y económicos, por parte de la autoridad ambiental local en este caso CORPOBOYACA, además se deberá diseñar, capacitar y poner en marcha programas de sustitución y reconversión de las actividades agropecuarias que se venían desarrollando con anterioridad al 16 de junio de 2011 y que se encuentren al interior del área de páramo delimitada, con el fin de garantizar de manera gradual la aplicación de la prohibición. Donde no estará permitido otorgar nuevos títulos mineros o suscribir nuevos contratos para la exploración y explotación de hidrocarburos, ni el desarrollo de nuevas actividades agropecuarias. Dentro de los tres (3) años siguientes a la delimitación, las autoridades ambientales deberán zonificar y determinar el régimen de usos del área de páramo delimitada. (Min Ambiente, 2011, p. 10).

Específicamente para el tema agrícola se citan textualmente de la resolución en su Artículo 4 directrices específicas para actividades agropecuarias:

- Se deben diseñar y poner en marcha programas de sustitución y reconversión de las actividades agropecuarias velando por la protección de los servicios eco sistémico del páramo. 
- El desarrollo de actividades agropecuarias deberá tener en cuenta las guías ambientales para el sector agrícola y pecuario expedidas por el Ministerio de Ambiente y Desarrollo Sostenible.

- Debe prestarse especial atención a aquellas actividades agropecuarias de subsistencia o que están llamadas a garantizar el mínimo vital de las comunidades ubicadas al interior del páramo, en la gradualidad de la reconversión evitando en todo caso una ruptura abrupta de las comunidades con su entorno y contribuyendo al mejoramiento de sus condiciones de vida.

- La planeación del desarrollo de las actividades deberá incorporar herramientas de planificación: predial y promover la conservación de la agro biodiversidad

\section{REALIDAD ECONÓMICA DEL MUNICIPIO DE AQUITANIA FRENTE A LAS RECOMENDACIONES PARA LA DELIMITACIÓN DE PÁRAMOS}

Para entender las problemáticas generadas por la delimitación del páramo Tota Bijagual Mamapacha, es necesario describir la realidad económica y social del Municipio de Aquitania.

Según la ficha de caracterización del DANE: “el municipio de Aquitania tiene 876 km2 de extensión, siendo el segundo municipio en extensión del departamento, además cuenta con una población de 15413 habitantes según censo de 2005 en su casco urbano esta el 41\% de la población y en el área rural el 59\%”(DANE, 2005).

El complejo TBM, tiene una extensión total de 151247.062 hectáreas, de las cuales 56025,77 hectáreas están dentro de jurisdicción de municipio correspondientes al 37.04\% 
del complejo. Es decir más de la mitad de la extensión del municipio se encuentra dentro del área de paramo.

La economía del Municipio se basa principalmente en las actividades agrícolas, siendo el cultivo de cebolla larga el principal renglón económico, lo cual ha permitido que sea el primer productor del país, con un área de cultivo superior a las 1.500 hectáreas, partiendo desde el Municipio alrededor de 400 toneladas diarias hacia diferentes partes del país para su comercialización y distribución, existen otros cultivos como arveja, papa, zanahoria y haba que también se desarrollan dentro del municipio (CorpoBoyacá, 2007).

La ganadería también es un sector de gran importancia dentro de la economía del municipio con cerca de 15.000 cabezas de ganado bovino registradas, actividad la cual se lleva a cabo en las veredas de Sisvaca, Maravilla, Momita, Hirba, Toquilla y Soriano, principalmente. En las demás veredas, los habitantes efectúan labores de pastoreo de bovinos, ovinos y caprinos.

Es importante tener en cuenta que aun cuando las intenciones del estado estén fundamentadas, y la expedición de dichas resoluciones se encuentre motivadas en los objetivos ambientales actuales, estas acciones generan problemáticas sociales, culturales y económicas en varias familias que por tradición desarrollan actividades económicas en dichas sectores. Dado lo anterior la propuesta que se presento por parte de CorpoBoyacá consiste en que el traslado y la reasignación de los campesinos y la detención de las actividades tanto de agricultura como de ganadería que se realizan allí se realicen de manera parcial. (CorpoBoyacá, 2007). 
Por parte de CORPOBOYACA, se realizo una propuesta en la que se expone como finalidad principal realizar un programa de sustitución y reconversión de las actividades agropecuarias, es decir que dentro de un plazo razonable se buscara que los territorios en los que las familias tradicionalmente realizaban sus actividades económicos vayan siendo sustituidos por otros que no estén dentro de la delimitación de paramos, y que sean aptos para estas actividades en especifico.

Como se sabe, esto no puede realizarse de manera inmediata ya que dichas actividades adelantadas requieren de una preparación especial de los terrenos, movilizaciones de quienes poseen viviendas cercanas a dichos lugares entre otros fenómenos secundarios que se derivan de la sustitución. Es importante tener en cuenta el impacto económico que los traslados puedan generar, y considerar que en la Resolución que se presento no se estipula si se asignara un presupuesto especial para corre con dichos gastos, o si por otra parte será el municipio quienes estén encargados de correr con los gastos que se generen. (CorpoBoyacá, 2017).

De igual manera, en lo referente al control de plagas y los productos específicos que son exigidos, así como la disposición adecuada de envases y empaques vacíos de los mismos, es evidente que este programa va a generar gastos tanto en su implementación como en las diferentes orientaciones que se le deben dar a los campesinos y cultivadores para que aprendan de qué forma deben utilizarse estos nuevos productos, gastos que no están siendo contemplados ni en la resolución ni tampoco en el momento de su ejecución, ya que no se ha determinado con certeza, como cuando y en qué lugar se desarrollaran dichas actividades que son indispensables para el cuidado de los páramos y así poder lograr las metas propuestas y además, para que los cultivadores puedan realizar sus actividades de 
manera correcta y sin enfrentarse a posteriores problemas jurídicos que se puedan presentar a causa de la ignorancia de las nuevas prácticas que fueron reguladas.

En lo referente a la protección de los suelos, lo que se busco fue implementar técnicas adecuadas de manejo que eviten la salinización, compactación, erosión, contaminación, y, en general, la perdida o degradación de los suelos de los terrenos que fueron determinados como paramos, pero en este caso también es necesario que se capacite a las personas que tienen contacto directo con la tierra, ya que como han desarrollado estas actividades durante décadas, tienen sus propios procedimientos adquiridos por tradición, es decir, que llevan años realizando las tareas de la manera en que les fue trasmitido y se presenta un choque entre las nuevas prácticas que deben ser implementadas y aquellas que han venido usando durante años.

Allí radica la importancia de una correcta implementación de programas de capacitación intensivos para llevar a este avance a las personas que habitan allí y así asegurar la conservación los humedales, nacimientos hídricos, las áreas de recarga hídrica los cuales se ven afectados principalmente por una incorrecta utilización de los recursos hídricos, por la falta de conocimiento de la manera correcta de realizar la extracción del agua lo cual deriva en la contaminación de varias de las fuentes hídricas más importantes que se encuentran en cercanías al paramo.

Para que lo anterior llegue a ser una realidad es necesario tener en cuenta que la implementación de las guías ambientales para el sector agrícola y pecuario expedidas por el Ministerio de Aambiente y Desarrollo Sostenible cumple un papel protagónico. Por lo que consideramos que se debe hacer una amplia socialización de las mismas, encabezada por 
funcionarios capacitados provenientes directamente del Ministerio de Aambiente y

Desarrollo Sostenible y que puedan explicar, en sus términos, a los campesinos cuales son las practicas que deben ser modificadas y de qué manera. Igualmente es importante que se les brinden las herramientas necesarias para que esta transición sea realizada, muchas veces dadas las condiciones que se viven en el campo los campesinos no tienen acceso a las tecnologías o a los implementos necesarios para la realización de las actividades según lo exige la ley. Esta problemática no debe ser ignorada ya que de allí radica el éxito real del programa o su fracaso.

Adicional a lo anteriormente expuesto consideramos que para que todo lo estipulado en la resolución pueda llegar a un éxito materia es necesario que se le dé especial atención a algunas de las actividades agropecuarias de subsistencia o que están llamadas a garantizar el mínimo vital de las comunidades ubicadas al interior del páramo, es decir, que se lleguen a tener en cuenta los dos factores en una misma medida, ya que es indispensable que se le garantice a la población que habita estas tierras y cuyo sustento sale de los terrenos que fueron delimitados como paramos que no quedaran a la deriva, paras que así exista la motivación necesario para implementar las practicas adecuadas de cuidado de los terrenos, conjuntamente a la planificación predial se deben promover la conservacion de la agro biodiversidad para que los habitantes nativos no sientan que se está realizando un abuso, si no que por el contrario consideren que son parte de un proyecto de conservación de la biodiversidad pero también de mejoramiento de sus condiciones. Lo que se hace evidente luego de esta investigación, es que muchos de los habitantes y productores de la zona necesitan que el Gobierno y las entidades ambientales más importantes del país les brinden las herramientas necesarias para poder trabajar sobre los lineamientos que indican las 
nuevas leyes, ya que sin ellos se hará imposible que se dé una implementación acertada de lo contenido en la resolución. Según lo dicho en la resolución: “A partir del 2011 está prohibido el desarrollo de actividades agropecuarias y por tanto la ampliación de la frontera agropecuaria" (Min Ambiente, 2011). Lo que inevitablemente afectara a nuevos productores a los sectores que deseaban iniciar a realizar estas actividades allí, para lo que consideramos que las autoridades pueden disponer otros terrenos útiles para estas actividades y así cubrir el déficit de trabajo que se presentara inevitablemente dentro de la población campesina-. También es importante tener en cuenta que La reconversión y sustitución de actividades agropecuarias son en su naturaleza procesos graduales, lo cual las hace convenientes ya que el cambio no será tan brusco para la vida de aquellas personas que acostumbrarían a desarrollar actividades en la cercanía de sus hogares., ya que aunque esta resolución con contempla expropìamientos se darán desplazamientos como consecuencia del cambio de lugar de trabajo. La reconversión y sustitución se logra necesariamente con la construcción de acuerdos entre las partes.

\section{CONCLUSIONES Y RECOMENDACIÓNES}

La Alcaldía municipal de Aquitania debe solicitarle a CORPOBOYACA programas y proyectos específicos de reconversión y sustitución para los dueños de predios dentro del área delimitada. Realizar amplia capacitación a la población, explicándoles los lineamientos

por parte del Ministerio de Ambiente y Desarrollo Sostenible con el fin de dar claridad al 
proceso que se va a llevar a cabo, teniendo como actores principales agricultores y pequeños ganaderos.

Solicitar a CORPOBOYACA, asesoramiento a la Alcaldía del Municipio con el fin de llevar a cabo la revisión general y actualización al esquema de ordenamiento territorial, para que los lineamientos queden plasmados el nuevo EOT

Solicitar al Ministerio de Agricultura acompañamiento para formulación y búsqueda de financiación de proyectos productivos que busquen la reconversión y sustitución de agricultura en área de paramo.

Como con luciones principales se obtuvieron que el éxito de lo contemplado en la resolución corresponda en una gran medida a los programas complementarios que las autoridades estáteles y ambientales decidan implementar para dar a conocer las nuevas técnicas que los nativos de dichas tierras deben implementar.

Además de la necesidad de que las sustituciones y reubicaciones se den de manera progresiva, para que dicha implementación no sea percibida como un ataque o como una violación a los derechos fundamentales si no que por el contrario sea percibida positivamente por la población del municipio de Aquitania. 


\section{REFERENCIAS}

CorpoBoyacá, (2007). Informe técnico social y económico. Recuperado de:

www.corpoboyaca.gov.co/informe tecnico-social-economico

Ministerio de Ambiente, (2011). Resolucion 937 de 2011. Recuperado de:

https://www.icbf.gov.co/cargues/avance/docs/resolucion_minambientevdt_0937_2011.htm

Ministerio de Ambiente, (2016). Resolución No. 1771 del 28 de Octubre 2016.

Recuperado de: www.corpochivor.gov.co/wpdm.../resolucion-1771-del-28-de-octubre-de2016/

Congreso de la Republica de Colombia (1991). Constitución Política de Colombia (1991) Recuperado de:

http://www.secretariasenado.gov.co/senado/basedoc/constitucion_politica_1991.html

Congreso de la Republica de Colombia (1993). Ley 99 de 1993. Recuperado de: http://www.secretariasenado.gov.co/senado/basedoc/ley_0099_1993.html

Congreso de la Republica de Colombia (2010). Ley 1382 de 2010. Recuperado de: http://www.funcionpublica.gov.co/eva/gestornormativo/norma.php? $i=38863$

Congreso de la Republica de Colombia (2011). Ley 1450 de 2011. Recuperado de: https://www.procuraduria.gov.co/portal/media/file/docs/ddr/CompiladoNormativo_Parte3.p df

Congreso de la Republica de Colombia (2015). Ley 1753 de 2015. Recuperado de: https://colaboracion.dnp.gov.co/CDT/Normograma/Ley\%201753\%20de\%202015.pdf

Corte Constitucional (2016). Sentencia Corte Constitucional C-035 de 2016. Recuperado de: http://www.corteconstitucional.gov.co/relatoria/2016/c-035-16.htm 
